Fig. 1. Solid line: Schwarzschield Potential.

The Effective Potential for $\lambda=0$ and $\epsilon=0.001$

Dashed line: $\tilde{E}=1$. Dotted line: $\tilde{E}=1.5$. Dot-dashed line: $\tilde{E}=2$.

Fig. 2. Solid line: Schwarzschield Potential.

The Effective Potential for $\lambda=90$ and $\epsilon=0.001$

Dashed line: $\tilde{E}=9$. Dotted line: $\tilde{E}=13$. Dot-dashed line: $\tilde{E}=22$.

Fig. 3. The curves represent the points at which the effective potential $\tilde{V}_{e f f}^{2}$, the scalar curvature $\mathrm{R}$ and the Kretschmann curvature invariant I diverge.

Dashed line: $\lambda=0$ and $\epsilon=0.001$. Solid line: $\lambda=0$ and $\epsilon=0.001$.

Fig. 4. The Scalar Curvature $R$ for $\epsilon=0.001$

Solid line: $\lambda=0$ and $\tilde{E}=9$

Dashed line: $\lambda=90$ and $\tilde{E}=22$

$\begin{array}{llllllllllll}-10 & -1 & 3 & 0.54 & 5 & \|\|_{1} & 6 & 71.5 & 8 & 92 & 10^{r} & \mathrm{r}\end{array}$




\title{
Schwarzschild Field with Maximal Acceleration Corrections
}

\author{
A. Feoli ${ }^{a, b}$, G. Lambiase ${ }^{a}$, G. Papini ${ }^{c *}$, G. Scarpetta ${ }^{a, d \dagger}$ \\ ${ }^{a}$ Dipartimento di Scienze Fisiche "E.R. Caianiello", Università di Salerno, Italia. \\ ${ }^{a}$ Istituto Nazionale di Fisica Nucleare, Sez. di Napoli. \\ ${ }^{b}$ Facoltà d'Ingegneria, Università del Sannio, Benevento, Italia \\ ${ }^{c}$ Dept. of Physics, University of Regina, Regina, Sask. S4S 0A2, Canada \\ ${ }^{d}$ International Institute for Advanced Scientific Studies, Vietri sul Mare (SA), Italia
}

\begin{abstract}
We consider a model in which accelerated particles experience lineelements with maximal acceleration corrections. When applied to the Schwarzschild metric, the effective field experienced by accelerated test particles contains corrections that vanish in the limit $\hbar \rightarrow 0$, but otherwise affect the behaviour of matter greatly. A new effect appears in the form of a spherical shell, external to the Schwarzschild sphere, impenetrable to classical particles.
\end{abstract}

PACS: 04.70.-s, 04.70.Bw

Keywords: Schwarzschild metric, Quantum Geometry, Maximal Acceleration

\footnotetext{
*E-mail: papini@cas.uregina.ca

†E-mail: Scarpetta@physics.unisa.it
} 
One of the main problems of modern theoretical physics is the unification of Quantum Mechanics and General Relativity. A very interesting step in this direction was taken by Caianiello [1] who interpreted quantization as curvature of the relativistic eight dimensional space-time tangent bundle $T M=M_{4} \otimes T M_{4}$, where $M_{4}$ is the usual flat space-time manifold of metric $\eta_{\mu \nu}$ and signature -2 . In this space the standard operators of the Heisenberg algebra are represented as covariant derivatives and the quantum commutation relations are interpreted as components of the curvature tensor. The Born reciprocity principle and, equivalently, the symmetry between configuration and momentum space representations of field theory, are thus automatically satisfied.

An interesting feature of Caianiello's model is that the proper accelerations of massive particles along their worldlines are normalized to an upper limit $\mathcal{A}_{m}$ [2], referred to as maximal acceleration (MA). In some works $\mathcal{A}_{m}$ is fixed by the Planck mass $m_{P}=\left(\frac{\hbar c}{G}\right)^{1 / 2}$ [3], [四] and is therefore a universal constant. A direct application of Heisenberg's uncertainty relations [5], [6], as well as the geometric interpretation of the quantum commutation relations, suggest, however, that the natural limit for the proper acceleration of any massive particle be fixed by the particle rest mass itself according to the relation $\mathcal{A}_{m}=2 m c^{3} / \hbar$. Its existence, which would automatically rid black hole entropy of ultraviolet divergences [7], [8], can be also surmised in the context of Weyl space [9] and of a geometrical analogue of Vigier's stochastic theory [10], or conjectured on diverse classical and quantum grounds [11], [3].

The notion of MA is linked to the extended nature of particles. The inconsistency of the point-particle concept for a relativistic quantum particle is discussed by Hegerfeldt [12] who shows that the localization of the particle at a given point at a given time enters in incurable conflict with causality.

The introduction of an invariant interval in the eight-dimensional spacetime tangent fiber bundle $T M$, may be also interpreted as a regularization procedure of the field equations, alternative to that in which space-time is quantized by means of a fundamental length, as in [13], where the twopoint and four point Green's functions of a massless scalar field theory are explicitely constructed and shown to lead to the disappearance of ultraviolet divergences at the one loop level. The advantage of Caianiello's proposal is to preserve the continuum structure of space-time.

MA has long been familiar in the classical context [14]. 
In a quantum relativistic context, the analysis of string propagation in cosmological backgrounds has revealed that accelerations higher than a critical value give rise to Jeans-like instabilities [15]. These occur [16] when the acceleration induced by the background gravitational field is large enough to render the two string extremities causally disconnected because of the Rindler horizon associated with their relative acceleration. The critical acceleration $a_{c}$ is determined by the string size $\lambda$ and is given by $a_{c}=\lambda^{-1}=(m \alpha)^{-1}$, where $\mathrm{m}$ is the string mass and $\alpha^{-1}$ the usual string tension.

Frolov and Sanchez [17] have then found that a universal critical acceleration $a_{c} \simeq \lambda^{-1}$ must be a general property of the strings.

In all these instances the critical acceleration is a consequence of the interplay of the Rindler horizon with the finite extension of the particle. In Caianiello's proposal the maximal proper acceleration is a basic physical property of all massive particles, which is an inescapable consequence of quantum mechanics [5], [6], and must therefore be included from the outset in the physical laws. This requires a modification of the metric structure of space-time. It leads, in the case of Rindler space, to a manifold with a non-vanishing scalar curvature and a shift in the horizon [18]. The cut-off on the acceleration is the same required in an ad hoc fashion by Sanchez in order to regularize the entropy and the free energy of quantum strings [19]. MA is also invoked as a necessary cut-off by McGuigan in the calculation of black hole entropy [B].

Applications of Caianiello's model include cosmology 叫, where the initial singularity can be avoided while preserving inflation, the dynamics of accelerated strings [20], the energy spectrum of a uniformly accelerated particle [18], the periodic structure as a function of momentum in neutrino oscillations [21] and the expansion of the very early universe [16]. The model also makes the metric observer-dependent, as conjectured by Gibbons and Hawking [22].

The extreme large value that $\mathcal{A}_{m}$ takes for all known particles makes a direct test of the model very difficult. Nonetheless a direct test that uses photons in a cavity has also been suggested [23]. More recently, we have worked out the consequences of the model for the classical electrodynamics of a particle [24], the mass of the Higgs boson [25] and the Lamb shift in hydrogenic atoms [26]. In the last instance the agreement between experimental data and MA corrections is very good for $H$ and $D$. For $\mathrm{He}^{+}$the agreement between theory and experiment is improved by $50 \%$ when MA corrections are included. MA effects in muonic atoms also appear to be measurable [27] 
In this paper we analyze the modifications produced by MA in the motion of a scalar particle in the Schwarzschild field.

As stated above, there is indeed a simple way to endow space-time with a causal structure in which proper accelerations are limited. It consists in replacing the usual Minkowski line element $d s^{2}=\eta_{\mu \nu} d x^{\mu} d x^{\nu}$ with the line element in the eight-dimensional space-time tangent bundle $T M$

$$
d \tau^{2}=\eta_{A B} d X^{A} d X^{B} \quad A, B=0, \ldots, 7,
$$

where

$$
\begin{gathered}
\eta_{A B}=\eta_{\mu \nu} \otimes \eta_{\mu \nu}, \\
X^{A}=\left(x^{\mu}, \frac{c^{2}}{\mathcal{A}_{m}} \frac{d x^{\mu}}{d s}\right) \quad \mu=0, \ldots, 3,
\end{gathered}
$$

$x^{\mu}=(c t, \vec{x})$ is the usual space-time four-vector and $d x^{\mu} / d s=\dot{x}^{\mu}$ the fourvelocity.

In order to write the equations of motion in ordinary four-dimensional space-time, an embedding procedure is required [18], the first step of a process of successive approximations, which leads to an effective four-dimensional space-time geometry, induced on $M_{4}$ by the metric $g_{A B}$ of $T M$ through the parametric equations which govern the embedding of $M_{4}$ in $T M$. Given the coordinates $\xi^{\mu}$, chosen to parametrize $M_{4}$, and assigned on $M_{4}$ the velocity field $\dot{x}^{\mu}(\xi)$, the eight equations $x^{\mu}=x^{\mu}(\xi)$ and $\dot{x}^{\mu}=\dot{x}^{\mu}(\xi)$ constitute the set of equations needed to represent $M_{4}$ as an embedded submanifold of $T M$. The metric $g_{\mu \nu}(\xi)$, locally induced, is then given by $g_{\mu \nu}(\xi)=$ $\eta_{\alpha \beta}\left(\frac{\partial x^{\alpha}}{\partial \xi^{\mu}} \frac{\partial x^{\beta}}{\partial \xi^{\nu}}+\frac{1}{\mathcal{A}_{m}^{2}} \frac{\partial \dot{x}^{\alpha}}{\partial \xi^{\mu}} \frac{\partial \dot{x}^{\beta}}{\partial \xi^{\nu}}\right)$.

The first order approximation introduced by this procedure consists in defining the velocity field $\dot{x}^{\mu}$ as the one obtained by solving the ordinary four-dimensional equations of motion. The invariant line element (1) can therefore be written in the form

$$
\begin{aligned}
d \tau^{2}= & \eta_{\mu \nu} d x^{\mu} d x^{\nu}+\frac{1}{\mathcal{A}_{m}^{2}} \eta_{\mu \nu} d \dot{x}^{\mu} d \dot{x}^{\nu}= \\
& =\left[1+\frac{\ddot{x}_{\mu} \ddot{x}^{\mu}}{\mathcal{A}_{m}^{2}}\right] \eta_{\mu \nu} d x^{\mu} d x^{\nu} .
\end{aligned}
$$

As a consequence one obtains mass-dependent corrections to the effective space-time geometry experienced by accelerated particles, which in general 
induce curvature, and give rise to a mass dependent violation of the equivalence principle. In the classical limit $\left(\mathcal{A}_{m}\right)^{-1}=\frac{\hbar}{2 m c^{3}} \rightarrow 0$ the terms contributing to the modification of the geometry vanish and one returns to the ordinary space-time geometry.

In the presence of gravity, we replace $\eta_{\mu \nu}$ with the corresponding metric tensor $g_{\mu \nu}$, a natural choice which preserves the full structure introduced in the case of flat space. We obtain

$$
d \tau^{2}=\left(1+\frac{g_{\mu \nu} \ddot{x}^{\mu} \ddot{x}^{\nu}}{\mathcal{A}_{m}^{2}}\right) g_{\alpha \beta} d x^{\alpha} d x^{\beta} \equiv \sigma^{2}(x) g_{\alpha \beta} d x^{\alpha} d x^{\beta}
$$

The four-acceleration $\ddot{x}^{\mu}=d^{2} x^{\mu} / d s^{2}$ appearing in the conformal factor of (3) is not a covariant quantity necessarily orthogonal to the four-velocity $\dot{x}^{\mu}$, as in Minkowski space. The justification for this choice lies primarily with the quantum mechanical derivation of $\mathcal{A}_{m}$ [5], [6] which applies to $\ddot{x}^{\mu}$, is Newtonian in spirit (it requires the notion of force) and is only compatible with special relativity. No extension of this derivation to general relativity has so far been given. The choice of $\ddot{x}^{\mu}$ in (3) is, of course, supported by the weak field approximation to $g_{\mu \nu}$ which, to first order, is entirely Minkowskian. Other relevant distinctions must be made. The model introduced is not intended to supersede general relativity, but only to provide a method to calculate the MA corrections to a Schwarzschild line element. The effective gravitational field introduced in (3) can not be easily incorporated in general relativity (it violates, for one, the equivalence principle). Nor are the symmetries of general relativity indiscriminately applicable to (3). For instance, the conformal factor is not an invariant, nor can it be eliminated by means of general coordinate transformations. The embedding procedure requires that it be present and that it be calculated in the same coordinates of the unperturbed gravitational background. It is useful to keep these distinctions in mind in what follows.

For convenience, the natural units $\hbar=c=G=1$ will be used below.

In order to calculate the corrections to the Schwarzschild field experienced by a particle initially at infinity and falling toward the origin along a geodesic, one must calculate the metric induced by the embedding procedure (2) to first order in the parameter $\mathcal{A}_{m}^{-2}$. On choosing $\theta=\pi / 2$, one finds the conformal 
factor produced by the embedding procedure

$$
\sigma^{2}(r)=1+\frac{1}{\mathcal{A}_{m}^{2}}\left[\left(1-\frac{2 M}{r}\right) \ddot{t}^{2}-\frac{\ddot{r}^{2}}{1-2 M / r}-r^{2} \ddot{\phi}^{2}\right],
$$

where $\ddot{t}, \ddot{r}$ and $\ddot{\phi}$ are given by the standard results [14]

$$
\begin{aligned}
\ddot{t}^{2} & =\frac{\tilde{E}^{2}}{(1-2 M / r)^{4}} \frac{4 M^{2}}{r^{4}}\left[\tilde{E}^{2}-\left(1-\frac{2 M}{r}\right)\left(1+\frac{\tilde{L}^{2}}{r^{2}}\right)\right], \\
\ddot{r}^{2} & =\left(-\frac{M}{r^{2}}+\frac{\tilde{L}^{2}}{r^{3}}-\frac{3 M \tilde{L}^{2}}{r^{4}}\right)^{2}, \\
\ddot{\phi}^{2} & =\frac{4 \tilde{L}^{2}}{r^{6}}\left[\tilde{E}^{2}-\left(1-\frac{2 M}{r}\right)\left(1+\frac{\tilde{L}^{2}}{r^{2}}\right)\right] .
\end{aligned}
$$

$M$ is the mass of the source, $\tilde{E}$ and $\tilde{L}$ are the total energy and angular momentum per unit of particle mass $m$. The conformal factor $\sigma^{2}(r)$ is then given by

$$
\begin{gathered}
\sigma^{2}(r)=1+\frac{1}{\mathcal{A}_{m}^{2}}\left\{-\frac{1}{1-2 M / r}\left(-\frac{3 M \tilde{L}^{2}}{r^{4}}+\frac{\tilde{L}^{2}}{r^{3}}-\frac{M}{r^{2}}\right)^{2}+\right. \\
\left.+\left(-\frac{4 \tilde{L}^{2}}{r^{4}}+\frac{4 \tilde{E}^{2} M^{2}}{r^{4}(1-2 M / r)^{3}}\right)\left[\tilde{E}^{2}-\left(1-\frac{2 M}{r}\right)\left(1+\frac{\tilde{L}^{2}}{r^{2}}\right)\right]\right\} .
\end{gathered}
$$

In order to make a direct comparison with the motion in Schwarzschild geometry possible, we adopt the same procedure and notations of Ch. 25 of [14]. The only exception is here represented by our choice of metric signature $(-2)$. Starting from the fundamental statement that the magnitude of the energy-momentum four-vector is given by the rest mass $m$ of the particle $g_{\alpha \beta} p^{\alpha} p^{\beta}=m^{2}$ and using the modified metric (3), one obtains

$$
\left(\frac{d r}{d \tau}\right)^{2}=\frac{1}{\sigma^{4}(r)}\left[\tilde{E}^{2}-\left(1-\frac{2 M}{r}\right)\left(\sigma^{2}(r)+\frac{\tilde{L}^{2}}{r^{2}}\right)\right] \equiv \tilde{E}^{2}-\tilde{V}_{e f f}^{2}(r),
$$

which corresponds to the equation

$$
\left(\frac{d r}{d \tau}\right)^{2}=\tilde{E}^{2}-\left(1-\frac{2 M}{r}\right)\left(1+\frac{\tilde{L}^{2}}{r^{2}}\right) \equiv \tilde{E}^{2}-\tilde{V}^{2}(r)
$$


of Ref. [14]. The analysis of the motion can therefore be given in term of

$$
\tilde{V}_{e f f}^{2}(r)=\tilde{E}^{2}-\frac{\tilde{E}^{2}}{\sigma^{4}(r)}+\frac{1}{\sigma^{2}(r)}\left(1-\frac{2 M}{r}\right)\left(1+\frac{\tilde{L}^{2}}{r^{2} \sigma^{2}(r)}\right) .
$$

In a more traditional approach one would write

$$
\frac{1}{2}\left(\frac{d r}{d \tau}\right)^{2}+\tilde{P}_{e f f}(r)=\omega
$$

where $\omega \equiv\left(\tilde{E}^{2}-1\right) / 2$ [14]. Substituting (8) into (10) one obtains $\tilde{P}_{\text {eff }}=$ $\frac{1}{2}\left(\tilde{V}_{e f f}^{2}(r)-1\right)$. It now is convenient to introduce the adimensional variable $\rho=r / M$ and the parameters $\epsilon=\left(M \mathcal{A}_{m}\right)^{-1}$ and $\lambda=\tilde{L} / M$. The complete expression for $\tilde{V}_{e f f}^{2}(\rho)$ is then

$$
\tilde{V}_{e f f}^{2}(\rho)=\tilde{E}^{2}\left\{1+\frac{1}{\sigma^{2}(\rho)}\left[-\frac{1}{\sigma^{2}(\rho)}+\frac{1}{\tilde{E}^{2}}\left(1-\frac{2}{\rho}\right)\left(1+\frac{\lambda^{2}}{\rho^{2} \sigma^{2}(\rho)}\right)\right]\right\} .
$$

Notice that $\tilde{V}_{e f f}^{2} \rightarrow \tilde{E}^{2}$ as $\rho \rightarrow 2$ and $\rho \rightarrow 0$. Moreover $\tilde{V}_{e f f}^{2} \rightarrow 1$ as $\rho \rightarrow \infty$. Plots of (11) for different values of $\tilde{E}$ show a characteristic step-like behaviour in the neighborhood of $\rho=2$ (Fig. 1 for $\lambda=0$, radial motion, Fig. 2 for $\lambda \neq 0$ ). Fig. 1 clearly shows that the effective and Schwarzschild potentials cannot be distinguished from each other for distances ranging from infinity to points very near the Schwarzschild radius, where $\tilde{V}_{e f f}^{2}$ acquires a marked dependence on the energy of the particle and develops a barrier which prevents the particle from reaching the Schwarzschild horizon. An expansion of (9) in the neighborhood of $\rho=2$ yields, in fact, the behaviour of the height of the potential barrier as $\tilde{V}_{e f f}^{2} \sim \tilde{E}^{2}+\frac{(\rho-2)^{4}}{4 \epsilon^{2} \tilde{E}^{4}}+O\left((\rho-2)^{5}\right)$ which, clearly, has the minimum $\tilde{E}^{2}$ on the horizon $\rho=2$. This term vanishes only in the limit $\tilde{E} \rightarrow \infty$ and/or in the limit $\epsilon \rightarrow \infty$ for which $\mathcal{A}_{m}$ or $M$ or both vanish and the problem becomes meaningless.

A second interesting consequence of the line element (3) is represented by the shift to the left in the horizon position in the case of radial motion, $\lambda=0$ (Fig. 1). The new horizon actually becomes a true singularity. Nevertheless, the potential barrier prevents incoming massive particles of energy $\tilde{E}$ from falling into the pit in the effective potential. The addition of MA effects does therefore produce, already to lowest order in $\mathcal{A}_{m}^{-2}$, a spherical shell 
of radius $2<\rho<2+\eta$, with $\eta \ll 1$ where, according to (7) and (8), the velocity of any incoming particle becomes imaginary. Such a shell is classically impenetrable and remains so at higher orders of approximation in $\mathcal{A}_{m}^{-2}$. The analogous occurrence of a classically impenetrable shell was derived by Gasperini as a consequence of the breaking of the local $S O(3,1)$ symmetry [29]. A generalization of the Schwarzschild metric to include MA corrections was also given in [21] for the two-dimensional problem of particles in hyperbolic motion in a Kruskal plane. As is known, these particles are static relative to Schwarzschild coordinates $(r=$ const). The classically impenetrable shell and shift in horizon also occur in this instance.

Fig. 2 shows that the scenario is similar to the previous case for particles with energy $\tilde{E}$ higher than the centrifugal potential barrier (dot-dashed line). If, on the contrary, the particle's energy is lower, its motion does not differ from the classical one because in the physically accessible region $\left(\tilde{V}^{2} \leq \tilde{E}^{2}\right)$ $\tilde{V}_{e f f}^{2}$ does not differ significantly from the Schwarzschild potential. The modifications induced in the region near the Schwarzschild radius, i.e. the infinite potential barrier for low values of $\tilde{E}^{2}$ (dashed line in Fig. 2), or the finite, but very high potential barrier for intermediate values of $\tilde{E}^{2}$ (dotted line in Fig. 2), have no influence on the particle motion, because they occur in a region precluded by the centrifugal barrier. The existence of bound orbits is assured by the fact that $\tilde{V}_{\text {eff }}$ and the Schwarzschild potentials reach the same value rapidly for $\rho>2$.

Fig 3 provides information about the localization of the singularities of the effective potential, according to the values of $\tilde{E}$ and $\lambda$. For radial motion (dashed line in fig. 3) and for increasing values of $\tilde{E}$ one meets one, than three and than again one singularity point, always located in the region internal to the event horizon. When the normalized angular momentum $\lambda$ differs from zero (solid line in fig. 3), there is a critical value of $\tilde{E}$ above which no singularities appear. Below this critical value, two singularity points appear on the left of the Schwarzschild radius. At even lower energies the singularities become four, two on the left and two on the right of the Schwarzschild radius.

The singularities can best be analyzed by calculating the scalar curvature $R(\rho) \equiv R_{\mu}^{\mu}$ and the Kretschmann curvature invariant $I \equiv R_{\alpha \beta \gamma \delta} R^{\alpha \beta \gamma \delta}$ for the metric (3). The explicit results are cumbersome and will be given elsewhere. We have however ascertained that the $\lim _{\epsilon \rightarrow 0} R(\rho)=0$, as it should, because for $\epsilon=0$ (3) yields the unmodified Schwarzschild metric. Moreover 
$\lim _{\rho \rightarrow 2} R(\rho)=0, \lim _{\rho \rightarrow 0} R(\rho)=0$ for $\lambda \neq 0, \lim _{\rho \rightarrow 0} R(\rho)=-54 \mathcal{A}_{m}^{2}$ for $\lambda=0$ indicating that there is no singularity at the origin. The behaviour of $M^{2} R(\rho)$ for $\epsilon=0.001$ and different values of $\lambda$ and $\tilde{E}$ is given in Fig. 4 for $\rho \geq 2$.

The metric modifications (3) discussed in the previous section give rise to a new, interesting effect represented by the presence of a spherical shell, external to the Schwarzschild sphere, that is forbidden to any classical particle. Its implications are clear: no massive particle can reach the Schwarzschild horizon. Would, however, such a shell prevent the formation of a black hole? Certainly yes if the model is correct and accretion of massive particles is a viable process of formation for black holes. One must however be cautious and keep in mind that the occurrence of the shell is essentially dynamical and that the procedure followed is perturbative.

Additional questions must also be considered.

i) Massive quantum tunneling through the shell $2<\rho<2+\eta$. Preliminary calculations based on spinless particles and the Klein-Gordon equation do not seem to favour this possibility, but a more detailed analysis is necessary before reaching definitive conclusions.

ii) Inflow at constant speed. One may assume that accumulation of matter in proximity of the shell would provide a viscous background to the motion of other incoming or outgoing matter. Particles moving with constant speed would experience a normal Schwarzschild field and fall into the pit for suitable values of $\tilde{L}$ and $\tilde{E}$. It may be difficult to realize this situation in proximity of a strong source in any realistic way, but, a priori, not impossible.

iii) Trasformation of matter into photons. The existence of large accelerations $\left(\ddot{x}_{\mu} \ddot{x}^{\mu} \sim 0.02 \mathcal{A}_{m}^{2}\right)$ in proximity of the shell would generate enormous streams of photons (and gravitons) by brehmsstrahlung. Substantial capture of photons by a large gravitational field would in time lead to black hole formation because massless particles see unmodified Schwarzschild fields. In this case formation would be only delayed and the whole process would resemble a gigantic engine of which photons (and possibly other massless particles) would at the same time be product and fuel.

The power radiated away by a single electron in proximity of the shell can be estimated from the formula [28], [24] $P=-\left(2 e^{2} / 3 c\right) \ddot{x}_{\mu} \ddot{x}^{\mu}$. An expansion of $\ddot{x}_{\mu} \ddot{x}^{\mu}$ in the neighborhood $r=2 M+\delta M$ of the shell yields $\ddot{x}_{\mu} \ddot{x}^{\mu} \sim-\delta^{4} \mathcal{A}_{m}^{-2} /\left(4 \tilde{E}^{4}\right)+O\left(\delta^{5}\right)$, and typically $\delta \sim 0.005$. The power emitted is therefore independet of the mass of the source. 
For $\tilde{E}=3$ one finds $P \sim 23.9 \mathrm{erg} / \mathrm{s}$. At the highest accelerations, $\ddot{x}_{\mu} \ddot{x}^{\mu} \sim 10^{-2} \mathcal{A}_{m}^{2}, P \sim 3.1 \cdot 10^{8} \mathrm{erg} / \mathrm{s}$, and the conversion rest mass energy into radiation becomes extremely efficient.

\section{Acknowledgments}

Research supported by NATO Collaborative Research Grant No. 970150, by Italian Ministero dell'Università e della Ricerca Scientifica, fund ex $40 \%$ and $60 \%$ DPR 382/80, and by the Natural Sciences and Engineering Research Council of Canada.

\section{References}

[1] E.R. Caianiello, La Rivista del Nuovo Cimento 15 n.4 (1992) and references therein.

[2] E.R. Caianiello, Lett. Nuovo Cimento 32 (1981) 65; E.R. Caianiello, S. De Filippo, G. Marmo, and G.Vilasi, Lett. Nuovo Cimento 34 (1982) 112.

[3] H.E. Brandt, Lett. Nuovo Cimento 38 (1983) 522; 39 (1984) 192; Found. Phys. Lett. 2 (1989) 39; 4 (1991) 523; 11 (1998) 265.

[4] E.R. Caianiello, M. Gasperini, G. Scarpetta, Class. Quantum Grav. 8 (1991) 659;

M. Gasperini, in "Advances in Theoretical Physics" ed. E.R. Caianiello, (World Scientific, Singapore, 1991), p. 77.

[5] E.R. Caianiello, Lett. Nuovo Cimento 41 (1984) 370.

[6] W.R. Wood, G. Papini and Y.Q. Cai, Il Nuovo Cimento B104, 361 and (errata corrige) 727 (1989).

[7] G. 't Hooft, Nucl. Phys. B256 (1985) 727;

L.Susskind, J. Uglam, Phys. Rev. D50 (1994) 2700.

[8] M. McGuigan, Phys. Rev. D50 (1994) 5225. 
[9] G. Papini and W.R. Wood, Phys. Lett. A170 (1992) 409; W.R. Wood and G. Papini, Phys. Rev. D45 (1992) 3617; Found. Phys. Lett. 6 (1993) 409; G. Papini, Mathematica Japonica 41 (1995) 81.

[10] J. P. Vigier, Found. Phys. 21 (1991) 125.

[11] A. Das, J. Math. Phys. 21 (1980) 1506; M. Gasperini, Astrophys. Space Sci 138 (1987) 387; M. Toller, Nuovo Cimento B102 (1988) 261; Int. J. Theor. Phys. 29 (1990) 963; Phys. Lett. B256 (1991) 215;

R. Parentani, R. Potting, Phys. Rev. Lett. 63 (1989) 945;

P. Voracek, Astrophys. Space Sci 159 (1989) 181;

B. Mashhoon, Physics LettersA143 (1990) 176;

V. de Sabbata, C. Sivaram, Astrophys. Space Sci. 176 (1991) 145; Spin and Torsion in gravitation, World Scientific, Singapore, (1994);

D.F. Falla, P.T. Landsberg, Nuovo Cimento B 106, (1991) 669;

A.K. Pati, Nuovo Cimento B 107 (1992) 895; Europhys. Lett. 18 (1992) 285.

[12] G.C. Hegerfeldt, Phys. Rev. 10 D (1974) 3320.

[13] J.C. Breckenridge, V. Elias, T.G. Steele, Class. Quantum Grav.12 (1995) 637.

[14] C.W. Misner, K.S.Thorne, J. A. Wheeler, Gravitation, W.H. Freeman and Company, S. Francisco, 1973.

[15] N. Sanchez and G. Veneziano, Nucl. Phys. 333 B, (1990) 253;

M. Gasperini, N. Sanchez, G. Veneziano, Nucl. Phys., 364 B (1991) 365; Int. J. Mod. Phys.6 A (1991) 3853.

[16] M. Gasperini, Phys. Lett. 258 B (1991) 70; Gen. Rel. Grav. 24 (1992) 219.

[17] V.P. Frolov and N. Sanchez. Nucl. Phys. 349 B (1991) 815.

[18] E.R. Caianiello, A. Feoli, M. Gasperini, G. Scarpetta, Int. J. Theor. Phys. 29 (1990) 131. 
[19] N. Sanchez, in "Structure: from Physics to General Systems" eds. M. Marinaro and G. Scarpetta (World Scientific, Singapore, 1993) vol. 1, pag. 118.

[20] A. Feoli, Nucl. Phys. B396 (1993) 261.

[21] E.R. Caianiello, M. Gasperini, and G. Scarpetta, Il Nuovo Cimento B105 (1990) 259.

[22] G. Gibbons and S.W. Hawking, Phys. Rev. D15 (1977) 2738.

[23] G. Papini, A. Feoli, and G. Scarpetta, Phys, Lett. A202 (1995) 50.

[24] A. Feoli, G. Lambiase, G. Papini, and G. Scarpetta, Nuovo Cimento 112B (1997) 913.

[25] G. Lambiase, G. Papini, and G. Scarpetta, Nuovo Cimento 114B (1999) 189. See also S. Kuwata, Il Nuovo Cimento 111B (1996) 893.

[26] G. Lambiase, G. Papini, and G. Scarpetta, Phys. Lett. 244A (1998) 349 .

[27] C.X. Chen, G. Papini, N. Mobed, G. Lambiase and G. Scarpetta, Nuovo Cimento 114B (1999) 199.

[28] L.D. Landau and E.M. Lifshitz, The Classical Theory of Fields (Pergamon Press, Oxford) 1969.

[29] M. Gasperini, Phys. Rev. D34 (1986) 2260. 